OPEN ACCESS

Edited by:

Fan Jin,

Zhejiang University, China

Reviewed by:

Tomoko Kawai,

Hiroshima University, Japan

Qingxue Zhang,

Sun Yat-Sen Memorial Hospital, China

*Correspondence:

Can Dai

daican@live.cn

Ge Lin

linggf@hotmail.com

Specialty section:

This article was submitted to Genetics of Common and Rare

Diseases,

a section of the journal

Frontiers in Genetics

Received: 02 April 2021 Accepted: 20 April 2021

Published: 19 May 2021

Citation:

Chen Y, Wang Z, Wu Y, He W, Du J, Cai S, Gong F, Lu G, Lin G and Dai C

(2021) Case Report: A Novel

Heterozygous ZP3 Deletion

Associated With Empty Follicle

Syndrome and Abnormal Follicular

Development

Front. Genet. 12:690070 doi: $10.3389 /$ fgene.2021.690070

\section{Case Report: A Novel Heterozygous ZP3 Deletion Associated With Empty Follicle Syndrome and Abnormal Follicular Development}

\author{
Yongzhe Chen ${ }^{1,2}$, Zesong Wang ${ }^{3}$, Yueren $\mathrm{Wu}^{3}$, Wenbin $\mathrm{He}^{4,5}$, Juan $\mathrm{Du}^{1,2,4,5,6}$, \\ Sufen Cai ${ }^{1,2,4,5}$, Fei Gong ${ }^{1,2,4,5,6}$, Guangxiu Lu ${ }^{3,4,5,6}$, Ge Lin $^{1,2,4,5,6 *}$ and Can Dai ${ }^{3,4,5 *}$ \\ ${ }^{1}$ School of Basic Medical Science, Central South University, Changsha, China, ${ }^{2}$ National Health Commission Key Laboratory \\ of Human Stem Cell and Reproductive Engineering, Central South University, Changsha, China, ${ }^{3}$ Department of Basic \\ Medicine, School of Medicine, Hunan Normal University, Changsha, China, ${ }^{4}$ Reproductive and Genetic Hospital of China \\ International Trust Investment Corporation Xiangya, Changsha, China, ${ }^{5}$ Clinical Research Center for Reproduction and \\ Genetics in Hunan Province, Changsha, China, ${ }^{6}$ National Engineering and Research Center of Human Stem Cell, Changsha, \\ China
}

Background: Empty follicle syndrome (EFS) is defined as the complete failure to retrieve oocytes after ovarian stimulation. Although several mutations in $Z P 1, Z P 2, Z P 3$, and LHCGR have been identified as genetic causes of EFS, its pathogenesis is still not well-understood.

Methods: Whole-exome sequencing (WES) was employed to identify the candidate pathogenic mutations, which were then verified by Sanger sequencing. A study in $\mathrm{CHO}-\mathrm{K} 1$ cells was performed to analyze the effect of the mutation on protein expression. Additionally, immunohistochemistry (IHC) staining was used to examine follicular development and zona pellucida (ZP) assembly in the ovary of an EFS patient.

Results: A novel heterozygous deletion in ZP3 (c.565_579del[p.Thr189_Gly193del]) was identified in the EFS patient. It was inherited dominantly and resulted in significant degradation of the ZP3 protein. Oocytes with degenerated cytoplasm and abnormal ZP assembly were observed in follicles up to the secondary stage, and many empty follicle-like structures were present.

Conclusion: We identified a novel ZP3 mutation that expands the mutational spectrum associated with human EFS. We also showed the abnormal follicular development and ZP assembly of the EFS patient with the heterozygous ZP3 mutation, which provides new insights into the pathogenesis of EFS.

Keywords: ZP3 mutation, empty follicle syndrome, zona pellucida, female infertility, follicular development

\section{INTRODUCTION}

Female infertility has become an increasingly prominent problem. Approximately 50 million women around the world suffer from infertility (Mascarenhas et al., 2012). Regular ovulation and healthy oocytes are the basis of human reproduction. With the development of in vitro fertilization (IVF) as a treatment for infertility, we have been able to retrieve oocytes and discover their defects in vitro. 
Empty follicle syndrome (EFS), first reported in 1986 (Coulam et al., 1986), is commonly defined as the complete failure to retrieve oocytes after ovarian stimulation for IVF, despite normal follicular development and appropriate serum estradiol level (Revelli et al., 2017). Follicle atresia and oocyte degeneration due to ovarian aging may be possible causes (Awonuga et al., 1998). In recent years, mutations in four genes, including $L H C G R$, $Z P 1, Z P 2$, and $Z P 3$, have been linked to EFS (Yuan et al., 2017; Chen et al., 2018; Dai et al., 2019a; Lu et al., 2019; Yang et al., 2020; Wang et al., 2021). Nevertheless, the pathogenesis of EFS, especially in patients with gene mutations, remains largely unknown.

During IVF treatment, cumulus-oocyte complexes (COCs), which consist of granulosa cells surrounding the centrally located oocyte, are recovered from the individual's follicular fluid (Veeck, 1988). Each oocyte is encapsulated in a zona pellucida (ZP), an extracellular matrix that plays important roles in the communication between oocyte and granulosa cells (GCs) (Fagbohun and Downs, 1990), fertilization (Pang et al., 2011; Avella et al., 2014), and protection of the early embryo (Hasegawa and Koyama, 2007).

The human ZP comprises four glycoproteins encoded by ZP1$Z P 4$ genes (Lefievre et al., 2004). In recent years, mutations in $Z P$ genes have been reported to be responsible for oocyte anomalies and female infertility (Yang et al., 2020). An autosomal recessive truncating variant in $Z P 1$ was identified in a consanguineous Chinese family, and this was suggested to explain infertility in females with abnormal oocytes lacking a ZP (Huang et al., 2014). Chen et al. was the first to identify a heterozygous recurrent missense mutation in $Z P 3$ as the cause of EFS. This mutation exerts its effect via a dominant negative effect on the interaction of $\mathrm{ZP}$ proteins, thereby possibly affecting $\mathrm{ZP}$ assembly, preventing communication between the cumulus cells and the oocyte, and eventually leading to oocyte degeneration (Chen et al., 2017). In the past 3 years, 25 ZP1 mutations, two ZP2 mutations, and two $Z P 3$ mutations were identified in patients with EFS (Dai et al., 2019a; Yang et al., 2020; Wang et al., 2021; Zhang et al., 2021).

In the present study, we identified a novel heterozygous ZP3 deletion associated with EFS and female infertility, which was inherited dominantly in a Chinese family. We also assessed the EFS patient's follicular development and ZP assembly by using ovarian sections and immunohistochemical staining (IHC).

\section{CASE PRESENTATION}

\section{Clinical Characterization}

A patient affected by EFS (Figures 1A,B) was recruited from the Reproductive and Genetic Hospital of CITIC-Xiangya. She was 37 years old, with a diagnosis of primary infertility for 7 years. She underwent three failed artificial insemination (AI) treatments and four cycles of ovarian stimulation and oocyte retrieval in another hospital, in which no oocytes but three empty COCs containing small ooplasm-like fragments without ZP structure were obtained (Table 1).

In our hospital, she received the fifth ovarian stimulation by the GnRH antagonist protocol. The rFSH/HMG was administered at a dose of 300 IU on day 2 of the menstrual cycle, and 300 IU per day for 8 days. Considering her history of recurrent failures and degenerated oocytes without ZP in the follicular aspirate, oocyte retrieval was performed by laparoscopy, upon identification of two follicles measuring $15.5 \mathrm{~mm}$ and $18.0 \mathrm{~mm}$ in diameters. Her serum estradiol level was $501 \mathrm{pg} / \mathrm{mL}$. Unfortunately, no oocyte was obtained (Table 1).

\section{Identification of ZP3 Mutation}

The recurrent EFS in the patient led us to consider a genetic cause. To identify the causative mutation, we initially performed whole-exome sequencing (WES) and variant analysis in the proband and her mother. The following mutations were considered as candidate mutations: (1) mutations detected in the proband but absent in her mother; (2) mutations with a minor allele frequency below $1 \%$ in three public databases (1000 Genomes, the ExAC, and gnomAD Browsers); (3) exonic nonsynonymous or splice site variants, or coding INDELs; and (4) mutations with high gene expression (reads per kilobase million $\geq 30$ ) in human oocytes in both the previously described (Yan et al., 2013) and in-house RNA sequencing data. These criteria led us to identify a heterozygous deletion c.565_579del [p.Thr189_Gly193del] in ZP3. This mutation is highly conserved among species (Figure 1D) and is located within the $\mathrm{ZP}$ domain (ZPD) of the ZP3 protein, a structural module essential for the polymerization and assembly of $\mathrm{ZP}$ proteins (Figure 1C) (Jovine et al., 2002). To confirm the c.565579del and exclude the possibility of a heterozygous deletion of a large fragment in $Z P 3$, the full-length ZP3 cDNA was amplified for Sanger sequencing using total RNA from the peripheral blood and primers: sense 5' - CCTCCTGCTCTGGGGTAGTA$3^{\prime}$ and antisense 5'- CTTTTATTCGGAAGCAGACACAG-3'. As a result, the proband and her father harbored the same heterozygous deletion c.565-579del, while both her mother and brother had the wild-type, suggesting that the deletion had been paternally transmitted (Figures 1A,B) and had not affected male fertility. The karyotype of the proband was 46, XX.

\section{Effects of ZP3 Mutation on Protein Expression}

To test the effect of this $Z P 3$ mutation, expression plasmids carrying wild-type or mutant $Z P 3 \mathrm{cDNA}$ were transfected into Chinese hamster ovary (CHO-K1) cells and immunoblot analyses were performed by using primary antibodies ZP3 (1:500; Santa Cruz Biotechnology, sc-25802) and GAPDH (1:3000; Beyotime, AF0006), respectively. Antibody binding was detected with HRP-conjugated goat anti-rabbit (1:2000; CWBIO, CW0103S, for primary antibody of ZP3) and goat anti-mouse (1:2000; CWBIO, CW0102S, for primary antibody of GAPDH) secondary antibodies. The expression level of the mutant ZP3 protein was significantly lower than that of the normal protein (Figure 1E), suggesting that the mutant protein was unstable and degraded. Moreover, in the experiment of co-transfection of wild-type and mutant plasmids, simulating heterozygous mutation in the patient, the total level of ZP3 protein was as low as in the cells transfected with the mutant plasmid (Figure 1E), indicating a dominant negative effect of the mutant ZP3. 
A

I

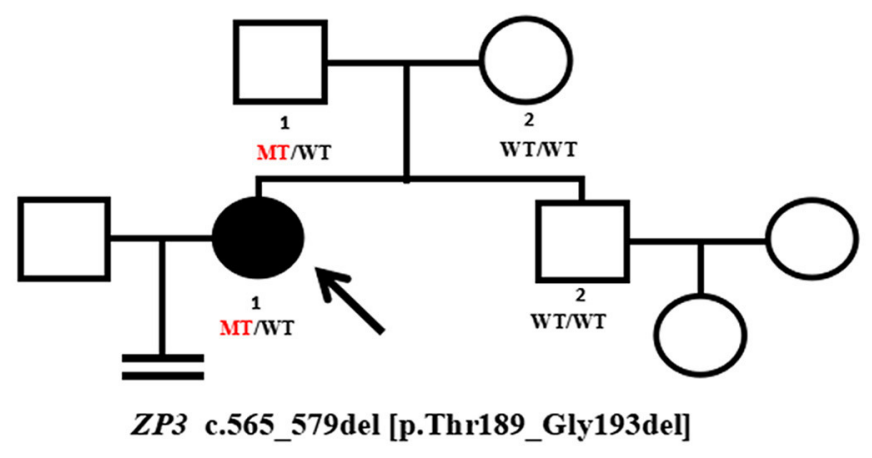

B

I-1

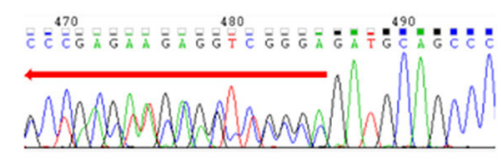

I-2

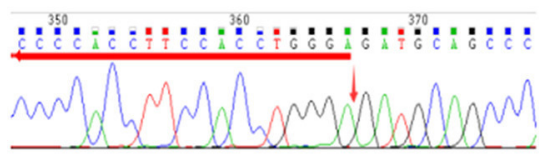

II-2

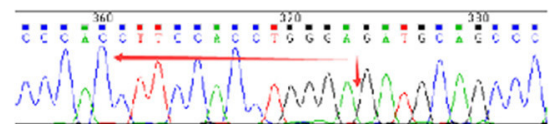

C

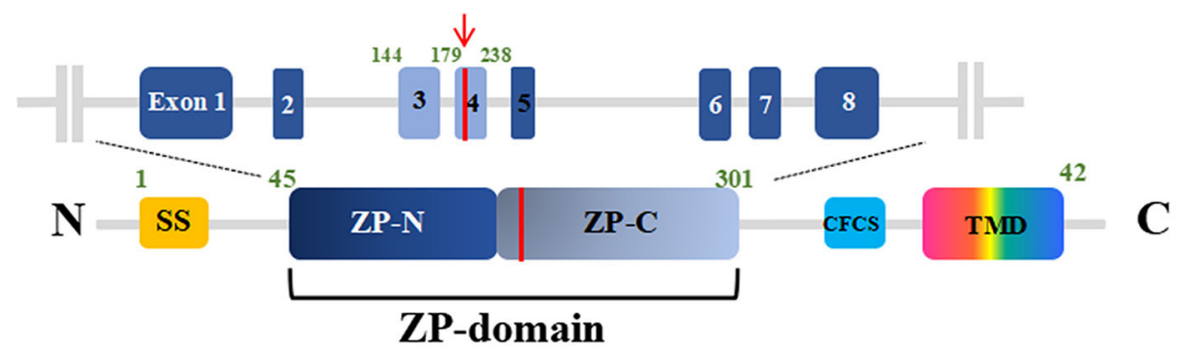

D

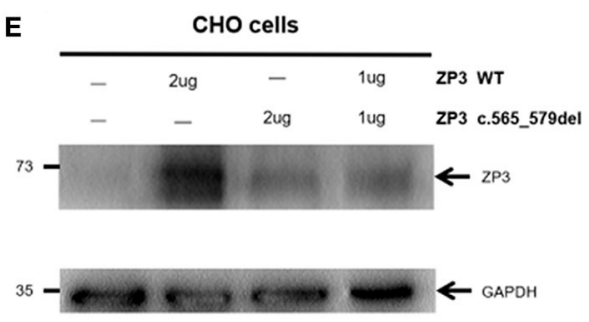

FIGURE 1 | Genotypic features of the EFS patient. (A) Pedigrees of the family with EFS and female infertility. The filled circle indicates the EFS patient. The arrow indicates the proband. The ZP3 genotype for each subject detected is shown, with WT indicating a normal allele and MT indicating the mutant allele. (B) Sanger genotype of ZP3 for each individual. (C) Location of the mutation (red line) is indicated in the genomic structure (top) and domain organization (bottom) of ZP3. SS, signals sequence; CFCS, consensus furin cleavage site; TMD, transmembrane domain. (D) Thr189_Gly193 is highlighted in red and conserved in the selected species. (E) Expression of wild-type (WT) and mutant ZP3 proteins in Chinese hamster ovary (CHO) cells examined by immunoblot analysis using anti-ZP3 antibodies.

\section{Follicular Development in the EFS Patient With ZP3 Mutation}

To examine follicular development and ZP assembly in the EFS patient carrying ZP3 mutation, IHC staining on the ovarian sections was performed. Control ovarian tissue was obtained from the ovarian wedge resection of an individual with polycystic ovary syndrome (PCOS). Antigen retrieval was performed using $0.01 \mathrm{M}$ sodium citrate buffer for $10 \mathrm{~min}$, then endogenous peroxidase activity was blocked with $0.3 \% \mathrm{H}_{2} \mathrm{O}_{2}$ in PBS for $15 \mathrm{~min}$, and non-specific binding sites were blocked with 5\% BSA in PBS at room temperature for $1 \mathrm{~h}$. Sections from the same follicle were then incubated overnight at $4^{\circ} \mathrm{C}$ with primary antibodies to VASA (1:100, Abcam, ab13840), ZP1 (1:100; Santa Cruz Biotechnology, sc-365435), and ZP3 (1:100; Santa Cruz Biotechnology, sc-398359), respectively. Antibody binding was detected with HRP-conjugated goat anti-rabbit (1:1000; Abcam, ab97051, for primary antibody of VASA) and goat anti-mouse (1:1000; Abcam, ab97245, for primary 
TABLE 1 | Clinical features of the EFS patient with a heterozygous ZP3 deletion.

\begin{tabular}{|c|c|c|}
\hline \multicolumn{3}{|c|}{ Basal hormones } \\
\hline $\mathrm{hFSH}$ & (3.03-8.08 mlU/mL) & 4.68 \\
\hline $\mathrm{hLH}$ & (1.80-11.78 mlU/mL) & 3.39 \\
\hline$E_{2}$ & $(21-251 \mathrm{pg} / \mathrm{mL})$ & 56 \\
\hline Prog & $(<0.1-0.3 \mathrm{ng} / \mathrm{mL})$ & 0.17 \\
\hline Testo & (0.38-1.97 ng/mL) & 0.56 \\
\hline PRL & (5.18-26.53 ng/mL) & 6.26 \\
\hline
\end{tabular}

\begin{tabular}{|c|c|c|c|c|c|}
\hline \multicolumn{6}{|c|}{ Clinical features with $\mathrm{COH}$ cycles } \\
\hline Cycle & 1 & 2 & 3 & 4 & 5 \\
\hline & (other center) & (other center) & (other center) & (other center) & (our center) \\
\hline Protocol & Ultra-long & Short & Ultra-long & GnRH antagonist & $\mathrm{GnRH}$ antagonist \\
\hline Initial dose of Gn (IU) & 225 & 225 & 225 & NA & 300 \\
\hline Duration of Gn days & 4 & 8 & 9 & NA & 8 \\
\hline \multirow[t]{2}{*}{ No. of COC retrieved } & 0 & 0 & 0 & 3 & 0 \\
\hline & & & \multicolumn{3}{|c|}{ Empty COCs with ooplasm-like fragments } \\
\hline
\end{tabular}

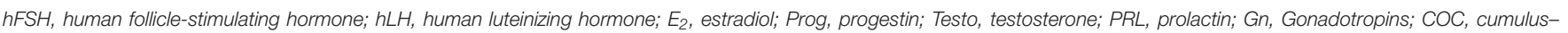
oocyte complex; NA, not available.

antibodies of ZP1 and ZP3) secondary antibodies. The sections were then developed using $\mathrm{DAB}$ kit and counterstained with hematoxylin. Using VASA staining, intact follicles containing an oocyte at the primordial to secondary stages were observed in the patient's ovary (Figures 2A-D). It is worth noting that, compared with homogeneous cytoplasm in normal oocytes (Figures 2P-AA), the patient's oocytes showed meshy cytoplasm (Figures 2D,I,N), which suggested that the oocytes might be undergoing degeneration. Using $\mathrm{ZP}$ staining, a continuous ZP structure increasing in width along with growing oocyte was observed in the normal control (Figures 2T-AA). In contrast, no clear and intact ZP structure was detected in the patient's secondary follicles (Figure 2N). Moreover, no follicles beyond secondary stage were found, but many empty follicle-like structures were detected (Figures 2E,J,O, 3). These results hint the abnormal ZP assembly and follicular development beyond the secondary stage in the EFS patient with ZP3 mutation.

\section{Discussion}

Since 2014, dozens of mutations in human $Z P$ genes have been identified as the cause of oocyte anomalies and female infertility. The mutations in $Z P$ gene are the predominant causes of EFS (Yang et al., 2020). Most ZP mutations in EFS patients have been identified in $Z P 1$, while only two were found in $Z P 2$ and $Z P 3$. Here, we performed genetic analysis on a Chinese woman who was diagnosed with primary infertility and EFS, considering that oocytes could not be obtained in five cycles of oocyte retrieval following ovarian stimulation. By using WES and Sanger sequencing, we identified a novel heterozygous deletion of $Z P 3$ inherited in an autosomal dominant pattern. This is in agreement with the earlier studies that identified two heterozygous missense mutations in ZP3 inherited dominantly in four independent EFS families. Our functional studies showed that this heterozygous ZP3 deletion led to the degradation of both the mutant and wild-type ZP3 proteins.

The human $\mathrm{ZP}$ is composed of four glycoproteins, called ZP1-4 (Lefievre et al., 2004). During folliculogenesis, ZP2 and $\mathrm{ZP}$, acting as building blocks, polymerize and incorporate into fibrils that form an extracellular matrix around the growing oocytes, while ZP1 acts as a cross-linker of the individual fibrils (Wassarman, 2008). ZPD is a common element of ZP proteins that consists of two separated domains marked as ZP-N and ZP-C. It is responsible for mediating homodimerization of intracellular ZP3 (dormant status) as well as the heteropolymerization of extracellular ZP2 and ZP3 and their incorporation into ZP fibrils (Bokhove and Jovine, 2018). Thr189_Gly193 is located within the ZP-C subdomain, and its deletion might affect formation and stabilization of dimeric ZP3, thereby leading to the degradation of ZP3 proteins within cells.

The zona matrix acts as a structural basis supporting the formation of gap junctions, a key structure for signal transduction between the growing oocyte and the surrounding cumulus cells (Wassarman, 2008). Our recent study presented the morphological evidence showing an abnormal ZP assembly and antral-follicular development in EFS patients with biallelic ZP1 mutations (Dai et al., 2019a). Here, similar anomalies in $\mathrm{ZP}$ assembly were observed in the EFS patient carrying a heterozygous ZP3 mutation, but degenerated oocytes were observed in the earlier stages and pre-antral follicles. However, dispersive ZP3 signals were detected surrounding the patient's oocyte within a secondary follicle (Figure $2 \mathrm{~N}$ ), which might result from secretion and accumulation of the relict ZP3 protein during follicle growth. We speculated that the shortage of secreted ZP3 protein and the defective ZPD in mutant ZP3 affect the ZP assembly, which further leads 


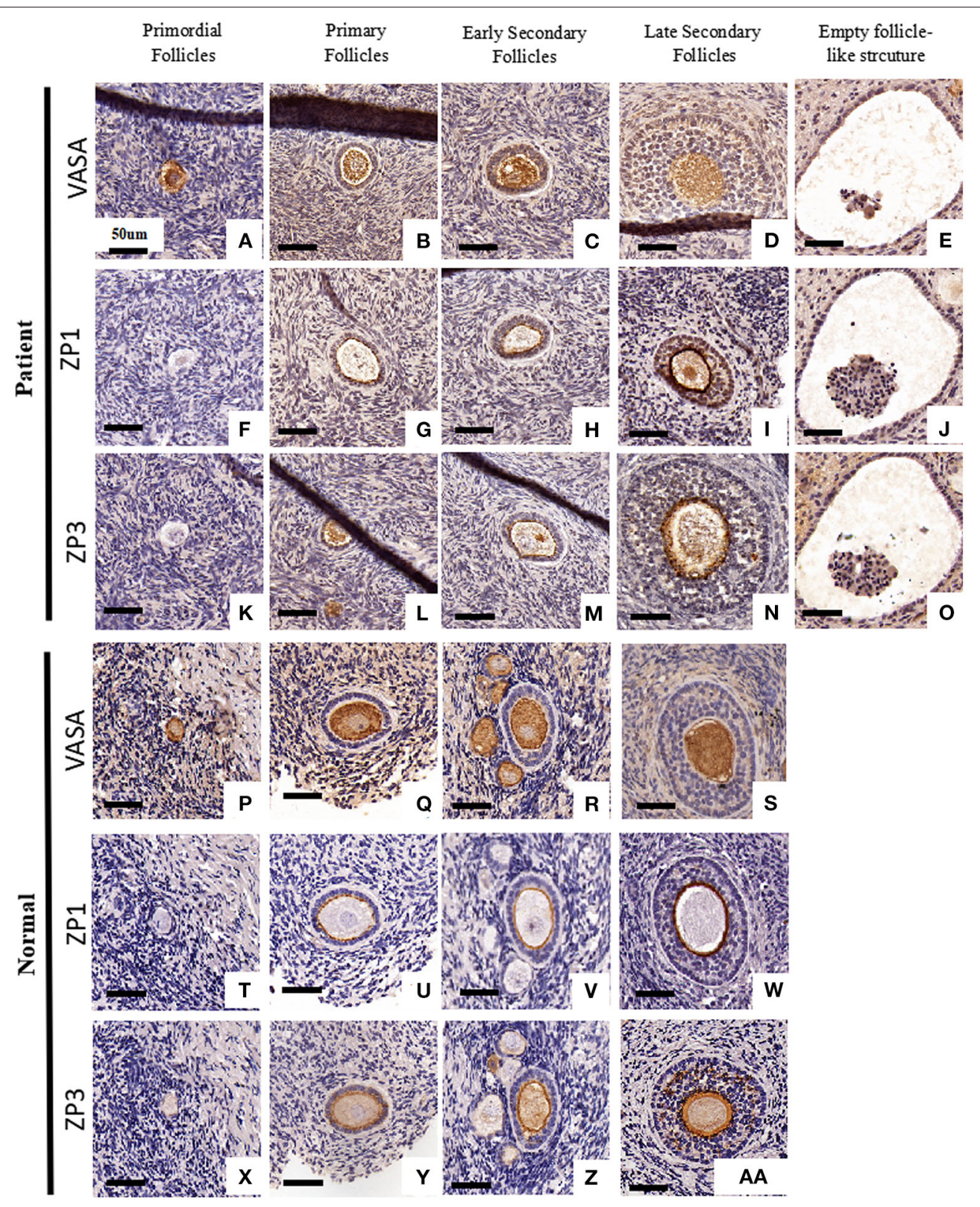

FIGURE 2 | Ovarian histology and immunohistochemistry staining of the EFS patient with a heterozygous ZP3 deletion. Ovaries isolated from the EFS patient carrying a ZP3 mutation (A-O) and a normal control (P-Z, AA) were sectioned and stained with antibodies against VASA (A-E, P-S), ZP1 (F-J, T-W), or ZP3 (K-O, X-Z, AA). The scale bars represent $50 \mu \mathrm{m}$.

to a defective gap junction and cumulus-oocyte complex organization, eventually resulting in oocyte degeneration and empty follicle-like structures.

Interestingly, our earlier study showed that patients carrying homozygous $Z P 2$-truncating mutations produced mature oocytes with a thin $\mathrm{ZP}$ that lacks ZP2, and this ZP2-null matrix sustained until the blastocyst stage (Dai et al., 2019b). ZP4 is a pseudogene in mice, while it is expressed in humans and may substitute ZP2 in forming a zona matrix as reported in mice
(Avella et al., 2014). Collectively, these observations suggest a key role of $\mathrm{ZP} 3$ in $\mathrm{ZP}$ assembly and support the opinion that the absence of ZP3 cannot be overcome (Fahrenkamp et al., 2020).

In conclusion, we reported a novel heterozygous $Z P 3$ deletion associated with EFS and female infertility. We also showed the abnormal ZP assembly and pre-antral follicular development. Our findings expand the mutational spectrum associated with human EFS, and provide new insights into its pathogenesis. 

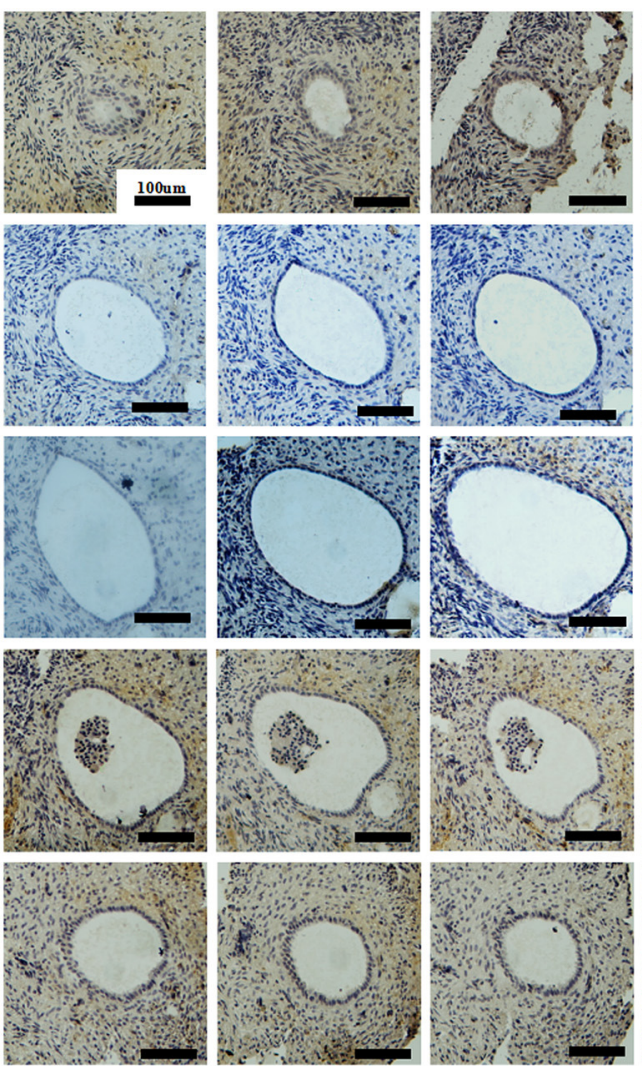
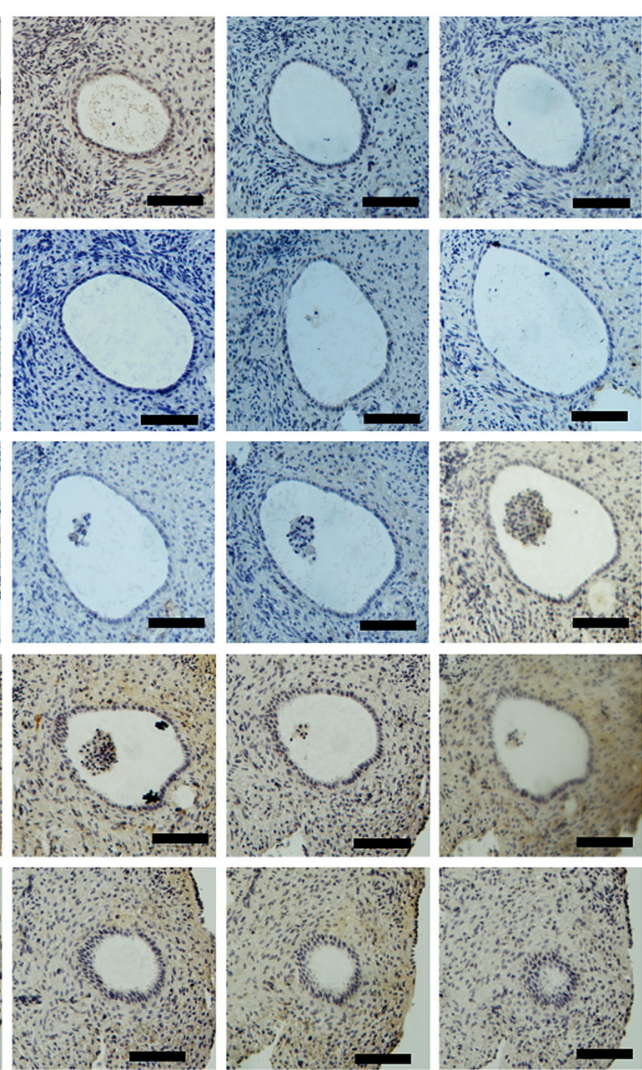

FIGURE 3 | Ovarian histology and immunohistochemistry staining of serial sections showing an intact empty follicle-like structure of the EFS patient with a heterozygous ZP3 deletion. There are a total of 30 sections with $5 \mu \mathrm{m}$ per section and $223 \mu \mathrm{m}$ in its widest part.

\section{DATA AVAILABILITY STATEMENT}

The raw data supporting the conclusions of this article will be made available by the authors, without undue reservation, to any qualified researcher.

\section{ETHICS STATEMENT}

The studies involving human participants were reviewed and approved by Ethics Committee of the Reproductive and Genetic Hospital of CITIC-Xiangya. The patients/participants provided their written informed consent to participate in this study.

\section{AUTHOR CONTRIBUTIONS}

$\mathrm{CD}, \mathrm{GLu}$, and GLin conceived and designed the study. YC, ZW, $\mathrm{WH}$, and $\mathrm{YW}$ carried out the experiments. JD, SC, and FG

\section{REFERENCES}

Avella, M. A., Baibakov, B., and Dean, J. (2014). A single domain of the ZP2 zona pellucida protein mediates gamete recognition in mice and humans. J. Cell Biol. 205, 801-809. doi: $10.1083 /$ jcb.201404025 provided the clinical samples. YC wrote the manuscript. CD and GLin critically commented on and edited the manuscript. All authors read and approved the final version of the manuscript.

\section{FUNDING}

This work was supported by grants from the National Key Research and Development Program of China (2016YFC1000200), the National Natural Science Foundation of China (81901553), and the Scientific Research Foundation of Reproductive and Genetic Hospital of China International Trust Investment Corporation (CITIC) Xiangya (YNXM-201911).

\section{ACKNOWLEDGMENTS}

We thank LetPub (www.letpub.com) for its linguistic assistance during the preparation of this manuscript.

Awonuga, A., Govindbhai, J., Zierke, S., and Schnauffer, K. (1998). Continuing the debate on empty follicle syndrome: can it be associated with normal bioavailability of beta-human chorionic gonadotrophin on the day of oocyte recovery? Hum. Reprod. 13, 1281-1284. doi: 10.1093/humrep/13. 5.1281 
Bokhove, M., and Jovine, L. (2018). Structure of zona pellucida module proteins. Curr. Top. Dev. Biol. 130, 413-442. doi: 10.1016/bs.ctdb.2018.02.007

Chen, C., Xu, X., Kong, L., Li, P., Zhou, F., and Zhao, S., et al. (2018). Novel homozygous nonsense mutations in LHCGR lead to empty follicle syndrome and 46, XY disorder of sex development. Hum. Reprod. 33, 1364-1369. doi: 10.1093/humrep/dey215

Chen, T., Bian, Y., Liu, X., Zhao, S., Wu, K., and Yan, L., et al. (2017). A recurrent missense mutation in ZP3 causes empty follicle syndrome and female infertility. Am. J. Hum. Genet. 101, 459-465. doi: 10.1016/j.ajhg.2017.08.001

Coulam, C. B., Bustillo, M., and Schulman, J. D. (1986). Empty follicle syndrome. Fertil. Steril. 46, 1153-1155. doi: 10.1016/S0015-0282(16)49898-5

Dai, C., Chen, Y., Hu, L., Du, J., Gong, F., and Dai, J., et al. (2019a). ZP1 mutations are associated with empty follicle syndrome: evidence for the existence of an intact oocyte and a zona pellucida in follicles up to the early antral stage. A case report. Hum. Reprod. 34, 2201-2207. doi: 10.1093/humrep/dez174

Dai, C., Hu, L., Gong, F., Tan, Y., Cai, S., and Zhang, S., et al. (2019b). ZP2 pathogenic variants cause in vitro fertilization failure and female infertility. Genet. Med. 21, 431-440. doi: 10.1038/s41436-0180064-y

Fagbohun, C. F., and Downs, S. M. (1990). Maturation of the mouse oocytecumulus cell complex: stimulation by lectins. Biol. Reprod. 42, 413-423. doi: 10.1095/biolreprod42.3.413

Fahrenkamp, E., Algarra, B., and Jovine, L. (2020). Mammalian egg coat modifications and the block to polyspermy. Mol. Reprod. Dev. 87, 326-340. doi: $10.1002 / \mathrm{mrd} .23320$

Hasegawa, A., and Koyama, K. (2007). Contribution of zona proteins to oocyte growth. Soc. Reprod. Fertil. Suppl. 63, 229-235. doi: 10.3192/jsirib.22.1

Huang, H. L., Lv, C., Zhao, Y. C., Li, W., He, X. M., and Li, P., et al. (2014). Mutant ZP1 in familial infertility. N. Engl. J. Med. 370, 1220-1226. doi: 10.1056/NEJMoa1308851

Jovine, L., Qi, H., Williams, Z., Litscher, E., and Wassarman, P. M. (2002). The ZP domain is a conserved module for polymerization of extracellular proteins. Nat. Cell Biol. 4, 457-461. doi: 10.1038/ncb802

Lefievre, L., Conner, S. J., Salpekar, A., Olufowobi, O., Ashton, P., and Pavlovic, B., et al. (2004). Four zona pellucida glycoproteins are expressed in the human. Hum. Reprod. 19, 1580-1586. doi: 10.1093/humrep/deh301

Lu, X., Yan, Z., Cai, R., Khor, S., Wu, L., and Sun, L., et al. (2019). Pregnancy and live birth in women with pathogenic LHCGR variants using their own oocytes. J. Clin. Endocrinol. Metab. 104, 5877-5892. doi: 10.1210/jc.2019-01276

Mascarenhas, M. N., Flaxman, S. R., Boerma, T., Vanderpoel, S., and Stevens, G. A. (2012). National, regional, and global trends in infertility prevalence since 1990: a systematic analysis of 277 health surveys. PLoS Med. 9:e1001356. doi: 10.1371/journal.pmed.1001356
Pang, P. C., Chiu, P. C., Lee, C. L., Chang, L. Y., Panico, M., and Morris, H. R., et al. (2011). Human sperm binding is mediated by the sialylLewis(x) oligosaccharide on the zona pellucida. Science 333, 1761-1764. doi: 10.1126/science. 1207438

Revelli, A., Carosso, A., Grassi, G., Gennarelli, G., Canosa, S., and Benedetto, C. (2017). Empty follicle syndrome revisited: definition, incidence, aetiology, early diagnosis and treatment. Reprod. Biomed. Online 35, 132-138. doi: 10.1016/j.rbmo.2017.04.012

Veeck, L. L. (1988). Oocyte assessment and biological performance. Ann. N. Y. Acad. Sci. 541, 259-274. doi: 10.1111/j.1749-6632.1988.tb22263.x

Wang, J., Yang, X., Sun, X., Ma, L., Yin, Y., and He, G., et al. (2021). A novel homozygous nonsense mutation in zona pellucida 1 (ZP1) causes human female empty follicle syndrome. J. Assist. Reprod. Genet. doi: 10.1007/s10815-021-02136-x. [Epub ahead of print].

Wassarman, P. M. (2008). Zona pellucida glycoproteins. J. Biol. Chem. 283, 24285-24289. doi: 10.1074/jbc.R800027200

Yan, L., Yang, M., Guo, H., Yang, L., Wu, J., and Li, R., et al. (2013). Single-cell RNA-Seq profiling of human preimplantation embryos and embryonic stem cells. Nat. Struct. Mol. Biol. 20, 1131-1139. doi: 10.1038/nsmb.2660

Yang, P., Chen, T., Liu, Y., Hou, Z., Wu, K., and Cao, Y., et al. (2020). The critical role of ZP genes in female infertility characterized by empty follicle syndrome and oocyte degeneration. Fertil. Steril. doi: 10.1016/j.fertnstert.2020.11.003. [Epub ahead of print].

Yuan, P., He, Z., Zheng, L., Wang, W., Li, Y., and Zhao, H., et al. (2017). Genetic evidence of "genuine" empty follicle syndrome: a novel effective mutation in the LHCGR gene and review of the literature. Hum. Reprod. 32, 944-953. doi: 10.1093/humrep/dex015

Zhang, D., Zhu, L., Liu, Z., Ren, X., Yang, X., and Li, D., et al. (2021). A novel mutation in ZP3 causes empty follicle syndrome and abnormal zona pellucida formation. J. Assist. Reprod. Genet. 38, 251-259. doi: $10.1007 / \mathrm{s} 10815-020-01995-0$

Conflict of Interest: The authors declare that the research was conducted in the absence of any commercial or financial relationships that could be construed as a potential conflict of interest.

Copyright $\odot 2021$ Chen, Wang, Wu, He, Du, Cai, Gong, Lu, Lin and Dai. This is an open-access article distributed under the terms of the Creative Commons Attribution License (CC BY). The use, distribution or reproduction in other forums is permitted, provided the original author(s) and the copyright owner(s) are credited and that the original publication in this journal is cited, in accordance with accepted academic practice. No use, distribution or reproduction is permitted which does not comply with these terms. 